18

\title{
Электрооптический метод исследования коагуляции нанодисперсных систем. Образование агрегатов частиц графита в водных электролитах
}

\author{
(C) О.С. Везо, А.В. Войтылов, В.В. Войтылов, М.П. Петров , А.А. Трусов \\ Физический фракультет Санкт-Петербургского государственного университета, \\ 198504 Петродворец, Санкт-Петербург, Россия \\ ฯ e-mail: m.p.petrov@spbu.ru
}

Поступила в редакцию 04.02.2020 г.

В окончательной редакции 04.02.2020 г.

Принята к публикации 19.02.2020 г.

\begin{abstract}
Продемонстрированы возможности электрооптического метода при изучении коагуляции жидких нанодисперсных систем, в частности коллоидов и суспензий. Использованы два электрооптических эффекта, зависящий, и не зависящий от поляризации света, проходящего сквозь систему в электрическом поле. Представлены результаты исследования кинетики коагуляции на ранних ее стадиях, связанных с образованием парных агрегатов из частиц графита, взвешенных в водных электролитах $\mathrm{AlCl}_{3}$ и $\mathrm{Th}\left(\mathrm{NO}_{3}\right)_{4}$. Показано, что системы устойчивы в широкой области концентрации электролитов и теряют устойчивость в узкой области, для которой электрокинетический потенциал частиц не превышает $5 \mathrm{mV}$. Показано, что определенные электрооптическим методом зависимости концентрации частиц от времени коагуляции в изоэлектрической точке хорошо согласуются с теорией быстрой коагуляции Смолуховского.
\end{abstract}

Ключевые слова: электрооптические эффекты, коагуляция, парные агрегаты, коллоиды и суспензии, частицы графита, статическое и динамическое светорассеяние.

DOI: $10.21883 /$ OS.2020.06.49401.22-20

\section{Введение}

Результаты исследования устойчивости и кинетики коагуляции дисперсных систем, содержащих наноразмерные частицы, имеют много приложений в науках об окружающей среде, коллоидах и суспензиях, материаловедении. Образование агрегатов из частиц в дисперсных системах меняет их оптические свойства, такие как мутность, индикатриса светорассеяния, показатель преломления. Нефелометрический метод широко используется при определении порога коагуляции и критической концентрации образования мицелл [1-4]. При образовании агрегатов из частиц изменяются индикатрисы светорассеяния, определяемые методами статического рассеяния, a также автокорреляционные функции интенсивности рассеянного света, определяемые методами динамического светорассеяния [5]. Для более детальных исследований коагуляции эффективен метод, основанный на одновременном изучении статического и динамического светорассеяния [6-9]. Он может быть использован при определении изменений размеров и концентрации частиц и агрегатов в процессе коагуляции. Аналогичные возможности предоставляют и электрооптические методы. Электрооптические измерения могут занимать крайне малое время (менее $1 \mathrm{~min}$ ), а релаксационные зависимости электрооптических эффектов могут быть использованы для определения функции распределения частиц и их агрегатов по размерам [10-12], которые меняются в процессе коагуляции. Это позволяет про- водить исследования в быстро коагулирующих системах. Если системы содержат частицы, размеры которых менее 20-30 nm, то в них легко наблюдать электрическое двойное лучепреломление [13]. Его описание аналогично описанию эффекта Керра в макромолекулярных растворах [14], но константа Керра существенно выше, чем в растворах, по причине более высокой степени ориентационного порядка частиц по сравнению с молекулами. Также появляется возможность изучать эффект, нелинейный по квадрату напряженности поля. При увеличении размеров частиц и появлении агрегатов из них в дисперсных системах инкремент показателя преломления систем снижается [15], и, как следствие, электрическое двойное лучепреломление становится слабовыраженным. Его теория до конца не построена [16], а экспериментальное наблюдение его осложнено другими, побочными эффектами [17]. В таких системах возрастает интенсивность светорассеяния и появляются электрооптические эффекты, обусловленные им. В проходящем свете наблюдаются электрический дихроизм, связанный как с поглощением, так и рассеянием света частицами [18], а также влияние электрического поля на коэффициент ослабления неполяризованного света дисперсными системами. Также наблюдается влияние поля на интенсивность и степень деполяризации света, рассеянного под углом $[19,20]$. В дисперсных системах, содержащих наноразмерные частицы алмаза, графита и нанотрубки углерода, наблюдались все вышеупомянутые эффекты [21-24]. 
Для исследованной нами системы, содержащей частицы графита, взвешенные в водном электролите, наблюдалось изменение дихроизма и константы мутности системы под воздействием электрического поля в процессе образования агрегатов из частиц. Цель данной работы продемонстрировать ряд возможностей электрооптического метода в задачах изучения коагуляции нанодисперсных систем, и изучить влияние многовалентных ионов в нанодисперсных системах графита на скорость их коагуляции.

\section{Методы}

\section{Теоретические основы электрооптического метода}

Для исследования кинетики коагуляции нанодисперсной системы графита был выбран метод, в котором исследовались изменения интенсивности света, прошедшего сквозь систему, возникающие при создании внешним электрическим полем ориентационной упорядоченности частиц и агрегатов из них. Величина и характерное время этих изменений интенсивности после выключения поля существенно зависят от процессов, связанных с образованием агрегатов из частиц.

Интенсивности лучей, прошедших сквозь дисперсную систему, в которой электрическим полем создана ориентационная упорядоченность частиц, и линейно поляризованных вдоль и ортогонально напряженности поля, можно представить в виде

$$
I_{\|, \perp}=I+A+\Delta I_{\|, \perp} .
$$

Подстрочные индексы $\|$ и $\perp$ здесь и далее относятся соответственно к свету, поляризованному вдоль и ортогонально напряженности внешнего поля. Здесь $I_{\|}$и $I_{\perp}-$ интенсивности прошедшего света при ориентационной упорядоченности частиц, а $I$ - при хаотической ориентации частиц и агрегатов в системе. Эти интенсивности определяются экспериментально. Все величины, входящие в (1), пропорциональны интенсивности падающего света, и их целесообразно определять как долю от интенсивности падающего света. Разности интенсивностей $I_{\|}-I$ и $I_{\perp}-I$ возникают при ориентационной упорядоченности частиц. Они содержат составляющую $A$, которая не зависит от поляризации света, и составляющие $\Delta I_{\|}$и $\Delta I_{\perp}$, которые зависят от поляризации света. Изменения $\Delta I_{\|}$и $\Delta I_{\perp}$ определяют дихроизм системы, обусловленный в дисперсных системах анизотропией поглощения и рассеяния света. Он наблюдается как в монохроматическом, так и в белом свете. При ориентационной упорядоченности частиц, имеющей ось симметрии вдоль напряженности поля, значения $\Delta I_{\|}$и $\Delta I_{\perp}$ взаимосвязаны [25] соотношением

$$
\Delta I_{\|}=-2 \Delta I_{\perp}
$$

В нефелометрических исследованиях определяется интенсивность I. В электрооптических исследованиях измеряются разности

$$
L_{\|}=I_{\|}-I \text { и } L_{\perp}=I_{\perp}-I .
$$

Значения $L_{\|}$и $L_{\perp}$ зависят от концентрации как частиц, так и агрегатов, образующихся в процессе коагуляции. Согласно теории коагуляции, концентрации частиц $n_{1}$ и парных агрегатов из них $n_{2}$ связаны [5,26-28] со скоростями их изменения соотношениями

$$
-\frac{d n_{1}}{d t_{1}}=k n_{1}^{2}, \frac{d n_{2}}{d t_{1}}=-\frac{1}{2} \frac{d n_{1}}{d t_{1}},
$$

где $t_{1}$ - время, отсчитанное от начала образования агрегатов, $k$ - константа скорости коагуляции. Соотношения (4) относятся к начальному этапу коагуляции, на котором агрегаты из нескольких частиц маловероятны, и их можно не учитывать. В монодисперсных системах со сферическими частицами константа $k$ зависит от произведения радиуса частиц на их константу поступательной диффузии, и, как следствие, не зависит от их размеров. В полидисперсных системах размеры частиц слабо влияют на скорость коагуляции, и соотношения (4) также применимы к полидисперсным системам, если различие размеров частиц в них не слишком велико. Величину дихроизма системы можно определить соотношением

$$
N=L_{\|}-L_{\perp}
$$

и, как следует из соотношений (1) и (3), величина $N$ не зависит от величины $A$. Если размеры частиц малы по сравнению с длинами световых волн, то $L_{\|}$и $L_{\perp}$, главным образом определяются величинами $\Delta I_{\|}$и $\Delta I_{\perp}$. В дихроизм могут вносить вклад как отдельные частицы, так и агрегаты из малых частиц, образующиеся в процессе коагуляции. Если размеры частиц велики по сравнению с длинами световых волн, то значения $L_{\|}$и $L_{\perp}$ мало отличаются от величины $A$, которую, учитывая (1) и (2), можно определить соотношением

$$
A=\frac{1}{3} I_{\|}+\frac{2}{3} I_{\perp}-I=\frac{1}{3} L_{\|}+\frac{2}{3} L_{\perp} .
$$

Значения $L_{\|}, L_{\perp}$ и $A$ пропорциональны концентрации частиц в системе и при образовании агрегатов они меняются.

Если концентрация частиц невелика, а их ориентационная упорядоченность, созданная электрическим полем, близка к насыщенной, то в процессе ее релаксации после выключения поля зависимости $N$ и $A$ от времени $t$ определяются [10] соотношениями

$$
\begin{aligned}
& N(t)=\int_{r 1}^{r 2} \Delta K \exp (-6 D(r)) \varphi_{N}(r) d r, \\
& A(t)=\int_{\rho 1}^{\rho 2} \Delta S \exp (-2 D(\rho)) \varphi_{A}(\rho) d \rho .
\end{aligned}
$$


Здесь $\varphi_{N}(r)$ и $\varphi_{A}(\rho)-$ функции распределения частиц и агрегатов по размерам $r$ и $\rho, D(r)$ и $D(\rho)$ - их константы вращательной диффузии, а $\Delta K$ и $\Delta S-$ оптические веса. Если падающий свет белый, а частицы и агрегаты соизмеримы с длинами световых волн $(r, \rho>20-30 \mathrm{~nm})$ и существенно рассеивают свет, то для разбавленных систем можно полагать, что $\Delta K$ и $\Delta S$ пропорциональны их поперечным сечениям и концентрациям [29]. В результате можно записать

$$
\Delta K=C_{N} n_{N} r^{2}, \Delta S=C_{A} n_{A} \rho^{2},
$$

где $n_{N}$ и $n_{A}-$ концентрации частиц и агрегатов, определяющих величины $N$ и $A$ соответственно, а $C_{N}$ и $C_{A}$ - коэффициенты. Входящие в соотношения (7) и (8) размеры $r$ и $\rho$ - это гидродинамические радиусы, которые связаны [30] с константами вращательной диффузии соотношениями

$$
D(r)=k_{B} T\left(8 \pi \eta r^{2}\right)^{-1}, D(\rho)=k_{B} T\left(8 \pi \eta \rho^{3}\right)^{-1} .
$$

Для тонкого диска диаметром $d$ эта константа $D(d)=k_{B} T\left(2 \eta d^{3}\right)^{-1}$, и если $D(r)=D(d)$, то $d \approx 2.32 r$. Здесь $\eta-$ вязкость дисперсионной среды, $k_{B}-$ постоянная Больцмана, $T-$ абсолютная температура.

Зависимости $N(t)$ и $A(t)$ можно экспериментально определить и использовать для расчета средних значений времен релаксации $\tau_{N}$ и $\tau_{A}$. Определим их соотношениями

$$
\frac{1}{\tau_{N}} \approx-\left\langle\frac{d \ln N(t)}{d t}\right\rangle \text { и } \frac{1}{\tau_{A}} \approx-\left\langle\frac{d \ln A(t)}{d t}\right\rangle .
$$

Так же как и в случае релаксации эффекта Керра и удельной поляризации [31], их можно связать со средними значениями констант вращательной диффузии соотношениями

$$
\tau_{N}^{-1}=6\langle D(r)\rangle \text { и } \tau_{A}^{-1}=2\left\langle D_{A}(\rho)\right\rangle .
$$

Значения $\tau_{N}$ и $\tau_{A}$ можно использовать при оценке скорости коагуляции. Их изменение характеризует изменение средних объемов частиц и агрегатов. При более детальном исследовании процесса коагуляции экспериментальные зависимости $N(t)$ и $A(t)$ также можно использовать для определения функций распределения частиц и агрегатов по размерам. А именно функции распределения с оптическим весом

$$
f_{N}(r)=\Delta K \varphi_{N}(r), f_{A}(\rho)=\Delta S \varphi_{A}(\rho)
$$

можно определять как решения интегральных уравнений (7) и (8) [12]. Аналогичный подход используется при анализе полидисперсности методом динамического светорассеяния. Функции $f_{N}(r)$ и $f_{A}(\rho)$ могут быть использованы при определении истинных функций распределения частиц и агрегатов по размерам $\varphi_{N}(r)=r^{-2} f_{N}(r)$ и $\varphi_{A}(\rho)=\rho^{-2} f_{A}(\rho)$. Здесь опущены нормировочные множители функций $\varphi_{N}(r)$ и $\varphi_{A}(\rho)$.

\section{Аппаратура для электрооптических исследований}

Для проведения электрооптических исследований коагуляции была использована экспериментальная установка, позволяющая определять релаксационные зависимости $N(t)$ и $A(t)$. Для этого экспериментально определяются $I_{\|}(t), I_{\perp}(t), I$ и используются соотношения (5) и (6). Она аналогична экспериментальным установкам, используемым при наблюдении эффекта Керра [30]. Отличие составляет то, что на пути луча между ячейкой Керра и фотоприемником отсутствует фазовая пластинка и анализатор, который скрещен с поляризатором, расположенным перед ячейкой. Световой луч создается светодиодом с непрерывным спектром излучения в диапазоне длин волн 320-800 nm, а поляризатором на лимбе задается направление линии поляризации света, падающего на ячейку. Для создания поля, ориентирующего частицы между электродами ячейки с исследуемой системой, используется генератор импульсов с варьируемой длительностью от $1 \mathrm{~ms}$ до $2 \mathrm{~s}$, создающий в ячейке напряженность в диапазоне от 0 до $2.5 \mathrm{kV} / \mathrm{cm}$. Исследования проводились при частоте поля в импульсе $50 \mathrm{kHz}$, что исключало поляризацию электродов в ячейке.

Для определения размеров частиц в приготовленной для исследования системе также был использован метод динамического светорассеяния. Для измерения автокорреляционной функции интенсивности светорассеяния использован стандартный прибор Photocor, а измерения проводились при длине световой волны $\lambda=638 \mathrm{~nm}$.

\section{Определение объемной доли частиц}

Объемная доля частиц $\Delta V / V$ в исследованной дисперсной системе определялась с использованием соотношения

$$
\frac{\Delta V}{V}=\frac{\delta-\delta_{0}}{\delta_{C}-\delta_{0}},
$$

в которое входят экспериментально определяемые плотности: частиц $-\delta_{C}$, дисперсной системы $-\delta$ и дисперсионной среды $-\delta_{0}$. Для определения указанных плотностей использован измеритель плотности DMA5000 .

\section{Определение электрокинетического потенциала}

Для изменения устойчивости дисперсных систем изменялся поверхностный электрический заряд частиц при варьировании концентрации электролита, в котором они взвешены. При анализе изменений плотности поверхностного заряда частиц могут быть использованы зависимости $\zeta$-потенциала частиц от концентрации ионов. Для определения $\xi$-потенциала использована стандартная методика, связанная с использованием светорассеяния для измерения электрофоретической скорости частиц. Для определения среднестатистических значений электрофоретической скорости использован Malvern Zetasiser (MADLS). В области изоэлектрической точки 


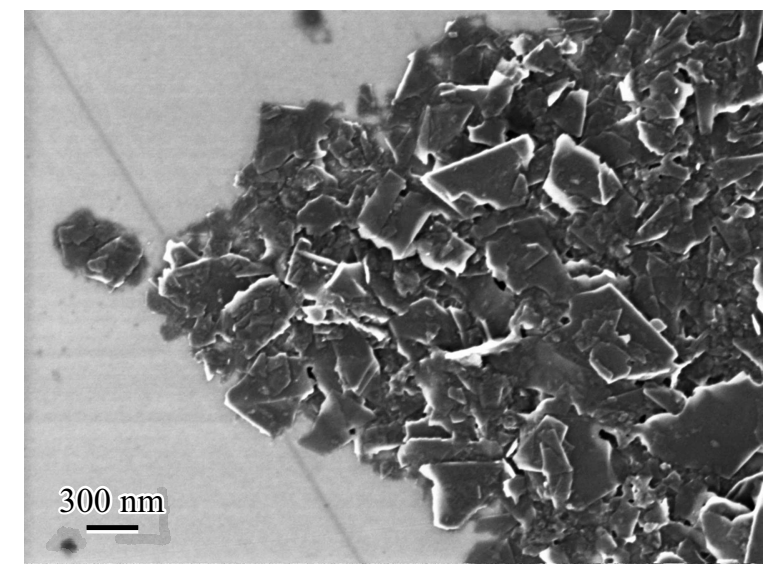

Рис. 1. Частицы графита.

разные частицы в системе могут иметь заряд разного знака, что влияет на скорость коагуляции исследуемой системы. При концентрациях электролита, близких к концентрации, отвечающей изоэлектрической точке, был использован микроэлектрофоретический метод, позволяющий определять электрофоретические подвижности отдельных частиц.

\section{Исследуемая дисперсная система}

Частицы графита были получены распылением графитового анода при помощи электрической дуги и стабилизированы лигносульфоновой кислотой. Далее приготавливалась высококонцентрированная водная суспензия, содержащая смесь частиц графита и бентонита. Разбавление водой и отстаивание суспензии позволило выделить дисперсную фазу графита без примеси бентонита. Для удаления поверхностно активных молекул и иных примесей с поверхности частиц графита дисперсная фаза прокаливалась, а многократные центрифугирование и ультразвуковая обработка водных взвесей с такой дисперсной фазой позволили ее отмыть и отделить слишком крупные и мелкие частицы, а также агрегаты частиц, которые мало пригодны для запланированных электрооптических исследований.

Согласно электронно-микроскопическим исследованиям частицы имели форму пластинок, размеры плоской части их поверхности лежали в диапазоне от 20 до $500 \mathrm{~nm}$, а наиболее вероятный размер был близок к $150 \mathrm{~nm}$. Здесь размер - это диаметр круга равновеликого плоской части поверхности частицы. Снимок частиц представлен на рис. 1. Для определения толщин частиц был использован метод напыления, которое производилось атомами вольфрама под углом $7^{\circ}$ к кварцевой подложке. Толщины исследованных частиц варьировали в диапазоне от 10 до $15 \mathrm{~nm}$.

Был проведен рентгеноструктурный анализ (XRD) полученной дисперсной фазы. Как следует из XRD спектров, дисперсную фазу составляла 2Н-модификация с незначительными добавками (менее 1\%) 3R-модификации. В спектрах, полученных методом рентгеновской фотоэлектронной спектроскопии (XPS), линии, отвечающие функциональным группам на поверхности частиц, не наблюдались. Были определены спектры комбинационного рассеяния света при длине волны падающего света $532 \mathrm{~nm}$. Они характеризовали поверхность и приповерхностные слои частиц графита, так как они существенно поглощают свет. Сухим частицам соответствовал спектр, содержащий основную линию первого порядка $\mathrm{G}\left(1586 \mathrm{~cm}^{-1}\right)$, линию второго порядка 2D $\left(2713 \mathrm{~cm}^{-1}\right)$ и линию D1 $\left(1357 \mathrm{~cm}^{-1}\right)$, соответствующую дефектам структуры графита. Отношение $\mathrm{G} / 2 \mathrm{D}=2.24$. Столь высокое значение этого отношения можно объяснить небольшим количеством оксидных групп на поверхности частиц. При исследовании тонкой структуры линии D1 других функциональных поверхностных групп не было выявлено. В спектре, соответствующем рассеянию света частицами в воде, наряду с линиями G, 2D и D1 наблюдается широкая полоса $\left(3150-3350 \mathrm{~cm}^{-1}\right)$, соответствующая $\mathrm{O}-\mathrm{H}$ группам, но не в воде, а на поверхности частиц.

Для проведения электрофоретических и электрооптических исследований была приготовлена базовая дисперсная система, которая содержала частицы данной дисперсной фазы в дистиллированной воде. Среднее расстояние между частицами на десятичный порядок превышало их размеры. Без добавок электролита базовая система была стабильна, и ее коэффициенты светорассеяния и экстинкции не менялись в течение 2-3 недель. Перед исследованиями кинетики коагуляции базовая система разбавлялась, определялась объемная доля частиц, далее к ней добавлялся электролит, понижающий устойчивость системы, и она подвергалась ультразвуковой обработке перед исследованием коагуляции.

\section{Результаты}

Были проведены исследования электрооптических свойств системы, содержащей частицы графита, взвешенные в воде. Соотношения (7)-(9) применимы к низкоконцентрированным системам, для которых значения электрооптических эффектов $N$ и $A$ меняются пропорционально концентрации частиц, предельно допустимое значение которой зависит как от оптических свойств частиц, так и от длины электрооптической ячейки, сквозь которую проходит световой луч. Это значение может уменьшаться при увеличении мутности системы в процессе коагуляции. Было экспериментально определено, что для исследованных систем при длине ячейки $5 \mathrm{~cm}$ допустимые концентрации частиц графита соответствовали их объемной доле $\Delta V / V$ менее $10^{-4}$. Насыщенной ориентации частиц вдоль напряженности поля и соответственно экстремальным значениям $N$ и $A$ соответствует поле с напряженностью $400 \mathrm{~V} / \mathrm{cm}$ и более. При добавлении электролитов это минимальное 

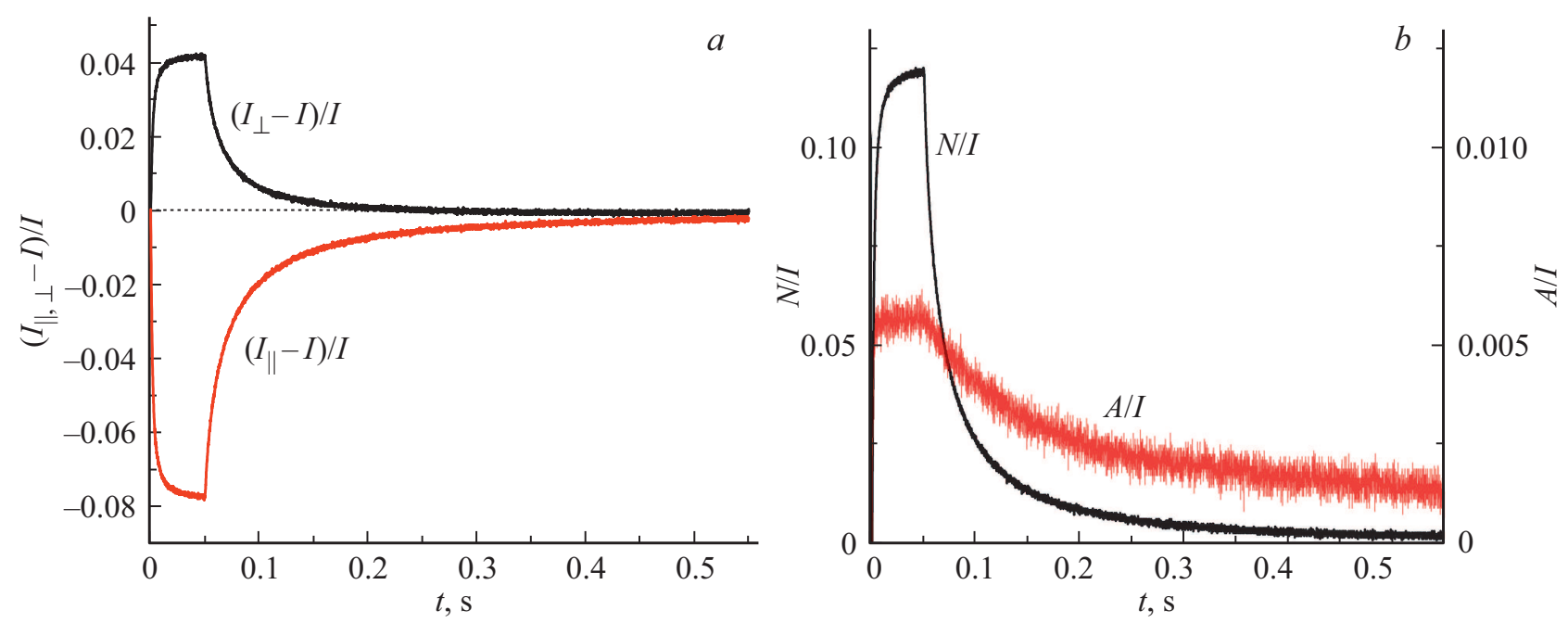

Рис. 2. Зависимости от времени: $(a)\left(I_{\|}-I\right) / I$ и $\left(I_{\perp}-I\right) / I,(b) N / I$ и $A / I$.

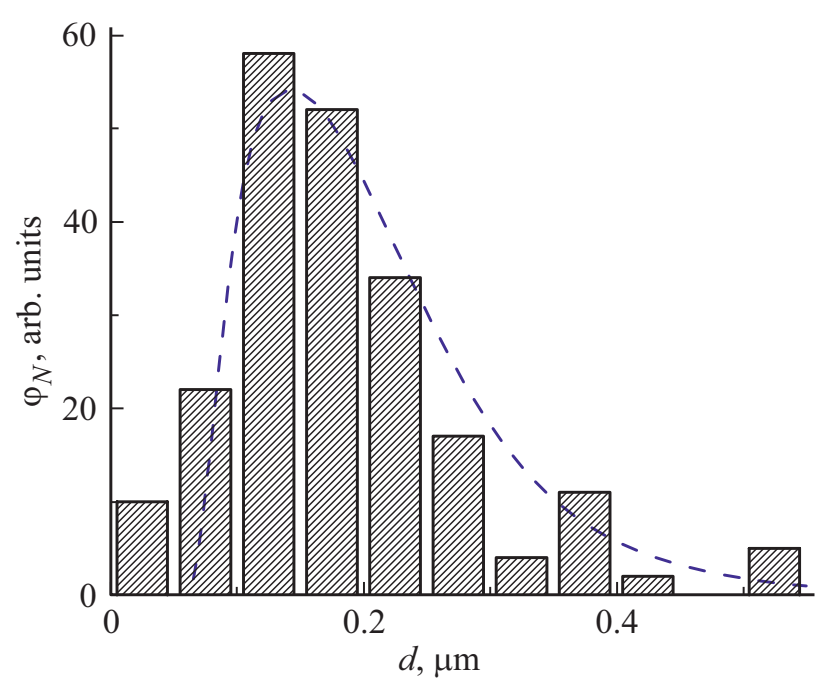

Рис. 3. Функция распределения $\varphi_{N}(d)$ (штриховая линия) и гистограмма.

значение могло повысится в 1.5-2 раза. На рис. 2 представлены зависимости от времени $t$ относительных изменений интенсивностей прошедшего света $\left(I_{\|}-I\right) / I$ и $\left(I_{\perp}-I\right) / I$, возникающих во время и после воздействия импульса синусоидального электрического поля частотой $50 \mathrm{kHz}$, эффективной напряженностью $400 \mathrm{~V} / \mathrm{cm}$ и длительностью $50 \mathrm{~ms}$ на систему, содержащую частицы графита в воде при их объемной доле $10^{-6}$.

Как можно видеть, эти изменения велики и электрооптические эффекты, связанные с изменением интенсивности света, прошедшего сквозь такую систему, ярко выражены даже при низких концентрациях частиц. На рисунке также представлены зависимости $N(t)$ и $A(t)$, из которых видно, что $N \gg A$ и $\tau_{A}>\tau_{N}$. Это свидетельствует о том, что для данной дисперсной системы эффект $A-$ это слабый побочный эффект и основной вклад в него вносят частицы более крупные, чем частицы, определяющие дихроизм $N$. Следует заметить, что для других дисперсных систем, частицы которых обладают менее выраженной анизотропией, значения $A$ близки к значениям $N$, а в ряде случаев превышают их. Релаксационная зависимость $N(t)$ и соотношение (7) были использованы для определения функции распределения $\varphi_{N}(d)$ частиц по их размерам в плоскости. Электронно-микроскопические снимки были использованы для определения гистограммы распределения частиц по размерам $d$. Функция $\varphi_{N}(d)$ и гистограммы представлены на рис. 3.

Как можно видеть, функция $\varphi_{N}(d)$ и гистограмма согласуются, что указывает на то, что основная доля частиц в системе вносит вклад в дихроизм. Частицы с размерами менее $50 \mathrm{~nm}$ не вносили свой вклад в наблюдаемые электрооптические эффекты. Следует учитывать, что поляризуемость таких частиц и анизотропия системы малы. Это не позволяло создать их заметную ориентационную упорядоченность, прикладывая к системе поле, которое необходимо для наблюдения эффектов $N$ и $A$. Также следует учитывать, что интенсивность света, рассеянного такими частицами, мала. Согласно представленной на рис. 3 гистограмме, доля таких частиц невелика, и ее можно не учитывать в начальной стадии коагуляции.

Для определения размеров частиц методом динамического светорассеяния не требуется их упорядоченной ориентации. Автокорреляционная функция интенсивности деполяризованного света $G_{H}^{V}(t)$ в основном определяется константами вращательной диффузии частиц [32], и характерные времена релаксации $\tau_{G}$ зависимости $G_{H}^{V}(t), \tau_{N}$ зависимости $N(t)$ должны быть близкими. Как показали исследования, зависимости $G_{H}^{V}(t)$ слабо меняются при изменении угла рассеяния и близки по форме к релаксационной зависимости $N(t)$. Время 


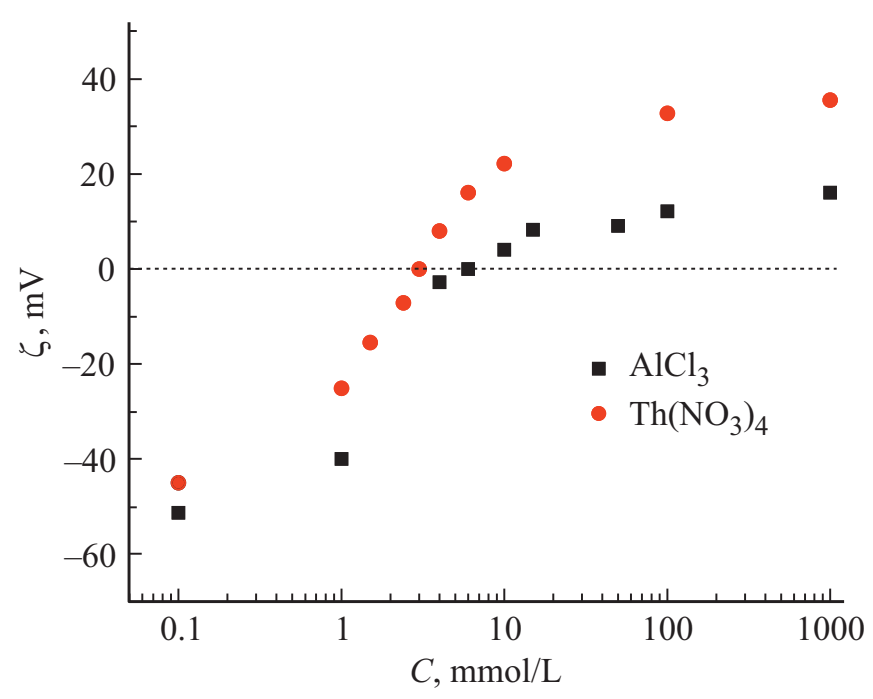

Рис. 4. Влияние концентрации $\mathrm{AlCl}_{3}$ и $\mathrm{Th}\left(\mathrm{NO}_{3}\right)_{4}$ на величину $\xi$-потенциала частиц графита.

релаксации $\tau_{G}$ определялось по формуле

$$
\frac{1}{\tau_{G}}=-\left\langle\frac{d G_{H}^{V}(t)}{d t}\right\rangle
$$

аналогичной формуле (10). Согласно результатам измерений $\tau_{N}=4 \mathrm{~ms}$, а $\tau_{G}=2.7 \mathrm{~ms}$ для зависимости $G_{H}^{V}(t)$, определенной при угле рассеяния $20^{\circ}$. Значения $\tau_{N}$ и $\tau_{G}$ пропорциональны третьей степени размеров частиц. По этой причине различие между средними размерами частиц, определенными этими методами, мало и не превышает $12 \%$.

Устойчивость дисперсных систем существенно зависит от поверхностного заряда частиц и, как следствие, их $\xi$-потенциала. В области изоэлектрической точки $(\zeta=0)$ электростатическое расталкивание между частицами отсутствует, а скорость коагуляции дисперсной системы максимально высокая для большинства коллоидов и суспензий. Если электролиты с ионами низкой валентности слабо меняют $\xi$-потенциал, то электролиты с трех- и четырехвалентными ионами существенно его изменяют, а изоэлектрическая точка достижима даже при слабых концентрациях многовалентных ионов в дисперсных системах.

Ниже представлены результаты электрофоретических и электрооптических исследований дисперсных систем, содержащих частицы графита, взвешенные в водных электролитах $\mathrm{AlCl}_{3}$ и $\mathrm{Th}\left(\mathrm{NO}_{3}\right)_{4}$. Концентрация электролитов $\mathrm{AlCl}_{3}$ и $\mathrm{Th}\left(\mathrm{NO}_{3}\right)_{4}$ в системах менялась от $10^{-7}$ до $10^{-3} \mathrm{~mol} / \mathrm{L}$.

Зависимости $\xi$-потенциала частиц графита от концентрации водных электролитов $\mathrm{AlCl}_{3}$ и $\mathrm{Th}\left(\mathrm{NO}_{3}\right)_{4}$ в дисперсных системах представлены на рис. 4. Этим зависимостям, так же как и представленным ниже результатам исследования, соответствовали объемная доля частиц $\Delta V / V=10^{-6}$, температура систем $21 \pm 0.5^{\circ} \mathrm{C}$.
Электрооптические исследования показали, что системы обладают высокой агрегативной устойчивостью за исключением узких зон коагуляции, в которых модуль $\zeta$-потенциала не превышал $5 \mathrm{mV}$. Вне зон коагуляции только через 2-3 недели значения $\tau$ начинали заметно возрастать, что свидетельствовало об образовании агрегатов из частиц. Были определены значения времени релаксации $\tau_{N}$ в зависимости от времени наблюдения $t_{1}$ при низких значениях $\xi$-потенциала. В изоэлектрической точке первые $5 \mathrm{~min} \tau_{N}$ менялось слабо, а далее возрастало практически линейно от логарифма времени $t_{1}$. Гидродинамический объем частицы или агрегата можно определить как объем сферы с константой вращательной диффузии частицы. Для определения среднего гидродинамического объема $b$ частиц и агрегатов, вносящих вклад в дихроизм, учитывая (10), можно использовать формулу $b=\tau_{N} k_{B} T / \eta$. При образовании агрегатов из частиц в процессе коагуляции объемы $b$ возрастают. Зависимости $b$ от времени $t_{1}$ агрегирования при разных значениях $\xi$-потенциала частиц представлены на рис. 5 .

Зависимости $N(t)$, соответствующие разным значениям времени агрегирования $t_{1}$ в изоэлектрической точке, и рассчитанные по ним функции $f_{N}(r)$ распределения частиц по их гидродинамическим радиусам представлены на рис. 6 и 7, а зависимости $A(t)$ и функции $f_{A}(\rho)$ представлены на рис. 8 .

В полидисперсных системах значения функций $\varphi_{N}(r)$ и $f_{N}(r)$ определяются счетной концентрацией отдельных частиц, если их размеры $r$ малы и можно полагать, что агрегатов таких размеров не образуется.

Если скорость коагуляции $k$, входящую в соотношение (4), считать не зависящей от размеров частиц, то функции $\varphi_{N}(r)$ и $f_{N}(r)$, изменяясь в процессе коагуляции, должны оставаться подобными между собой в этой области размеров $r$. Счетная концентрация частиц в системе $n_{1}$ и значения функций $\varphi_{N}(r)$ и $f_{N}(r)$ в области малых размеров должны быть связаны равенством

$$
\frac{1}{n_{1}} \frac{d n_{1}}{d t_{1}}=\frac{1}{\varphi_{N}(r)} \frac{d \varphi_{N}(r)}{d t_{1}}=\frac{1}{f_{N}(r)} \frac{d f_{N}(r)}{d t_{1}} .
$$

Представленные на рис. 6 и 7 зависимости $f_{N}(r)$ подобны при $r<200 \mathrm{~nm}$. Исключение составляют функции $f_{N}(r)$, соответствующие времени $t_{1}=1072$ и $2703 \mathrm{~min}$. При этих значениях $t_{1}$ доля агрегатов в области размеров $r<200 \mathrm{~nm}$ становится существенной. Зависимость концентрации частиц в системе от времени, определяемая интегрированием уравнения (4), может быть представлена соотношением

$$
\frac{n_{1}\left(t_{1}\right)}{n_{0}}=\frac{1}{1+t_{1} / T_{C}}
$$

которое можно экспериментально проверить, учитывая соотношение (11). Здесь $n_{0}-$ концентрация частиц до начала коагуляции, $T_{C}-$ период коагуляции. Такие зависимости, описывающие изменения концентрации частиц при коагуляции исследуемой дисперсной системы 

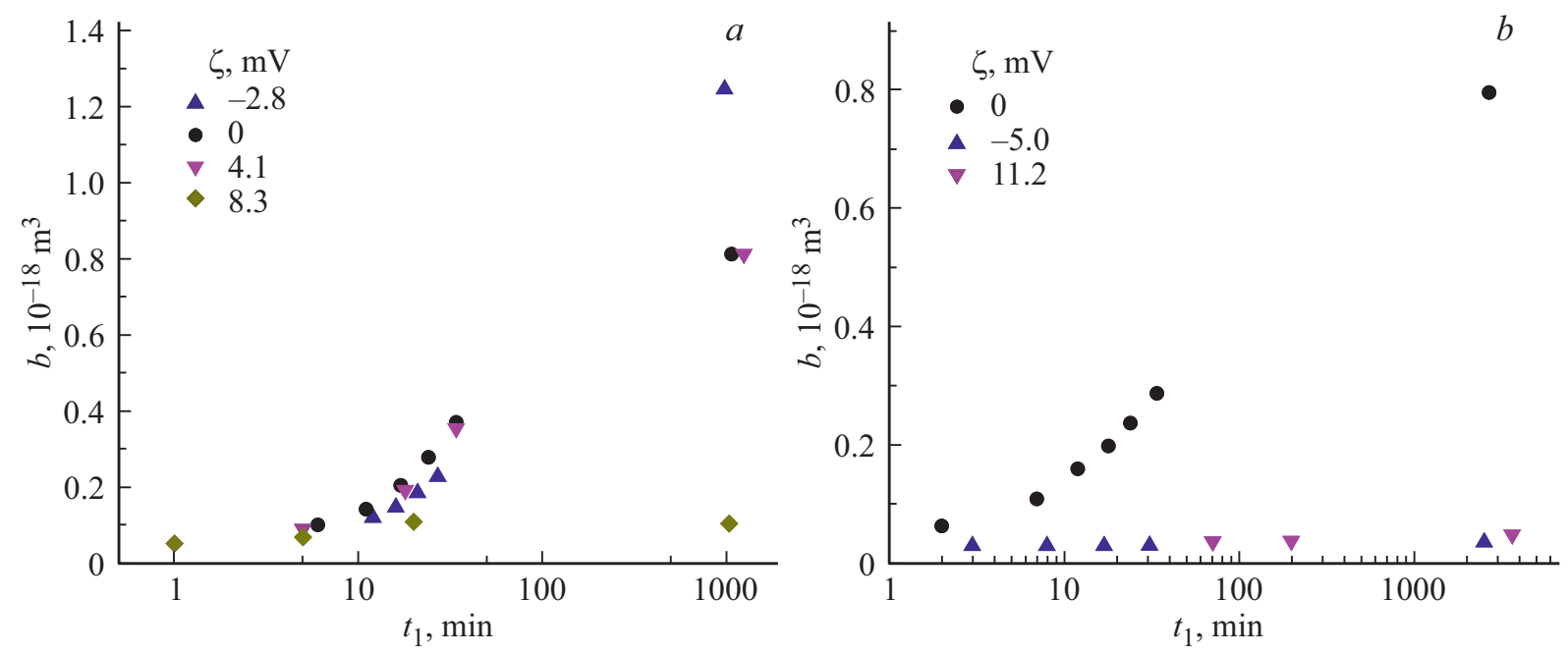

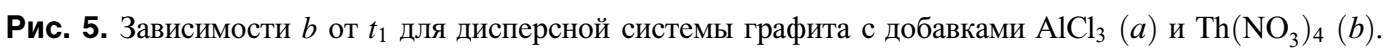
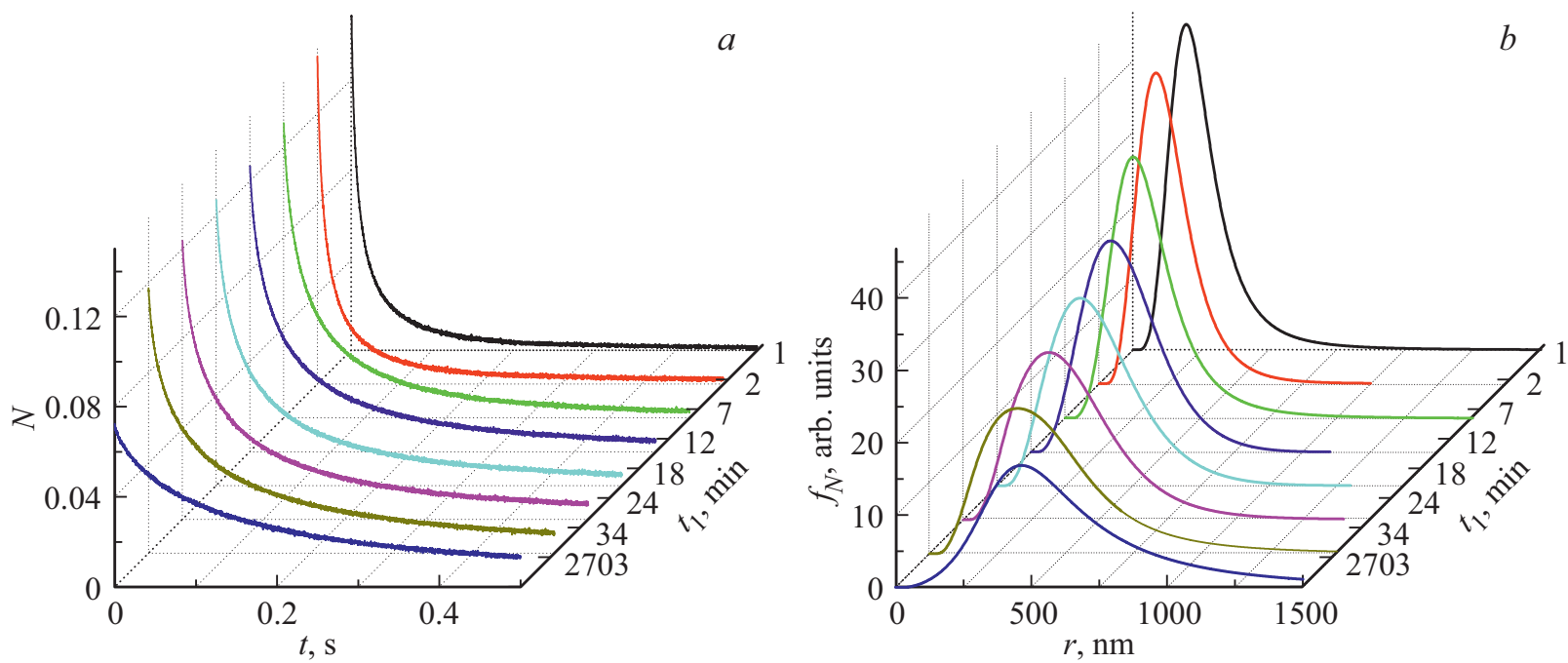

Рис. 6. Изменение со временем $t_{1}$ релаксационной зависимости $N(t)(a)$ и функции распределения $f_{N}(r)(b)$ для дисперсной системы графита с концентрацией электролита $\mathrm{Th}\left(\mathrm{NO}_{3}\right)_{4}$, соответствующей изоэлектрической точке.
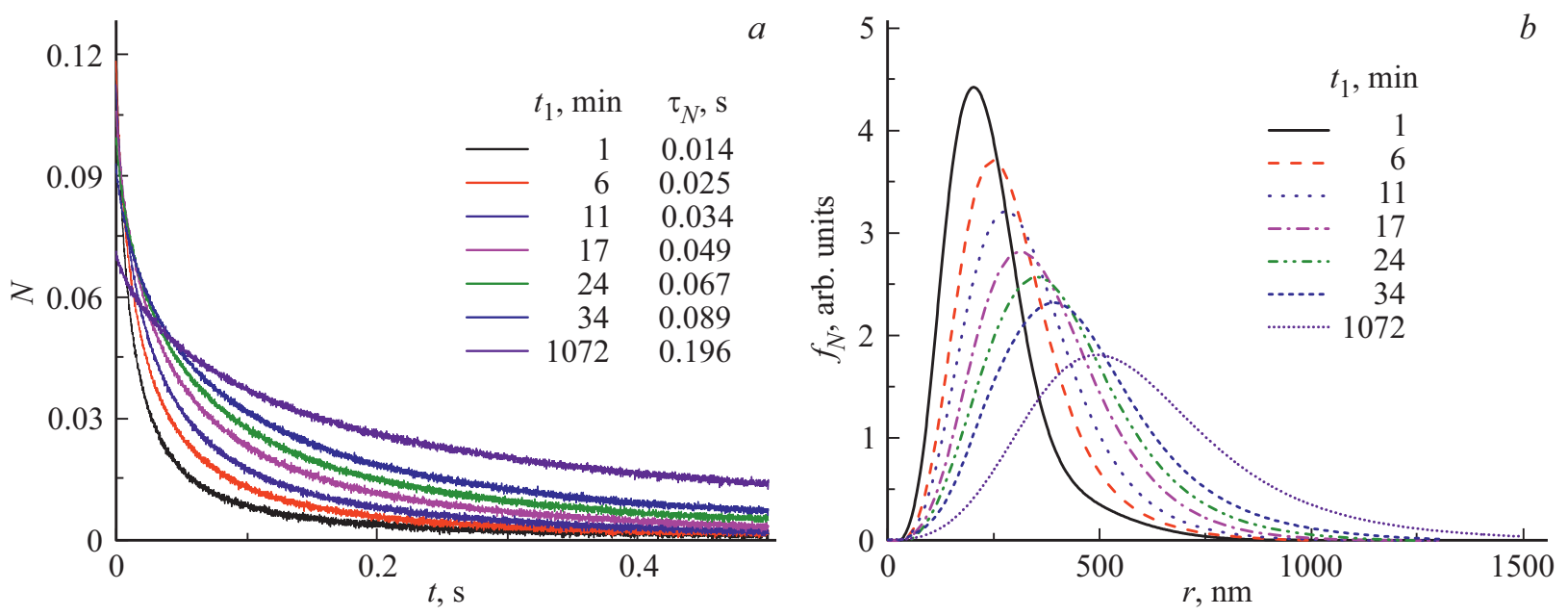

Рис. 7. Изменение со временем $t_{1}$ релаксационной зависимости $N(t)(a)$ и функции распределения $f_{N}(r)(b)$ для дисперсной системы графита с концентрацией электролита $\mathrm{AlCl}_{3}$, соответствующей изоэлектрической точке. 

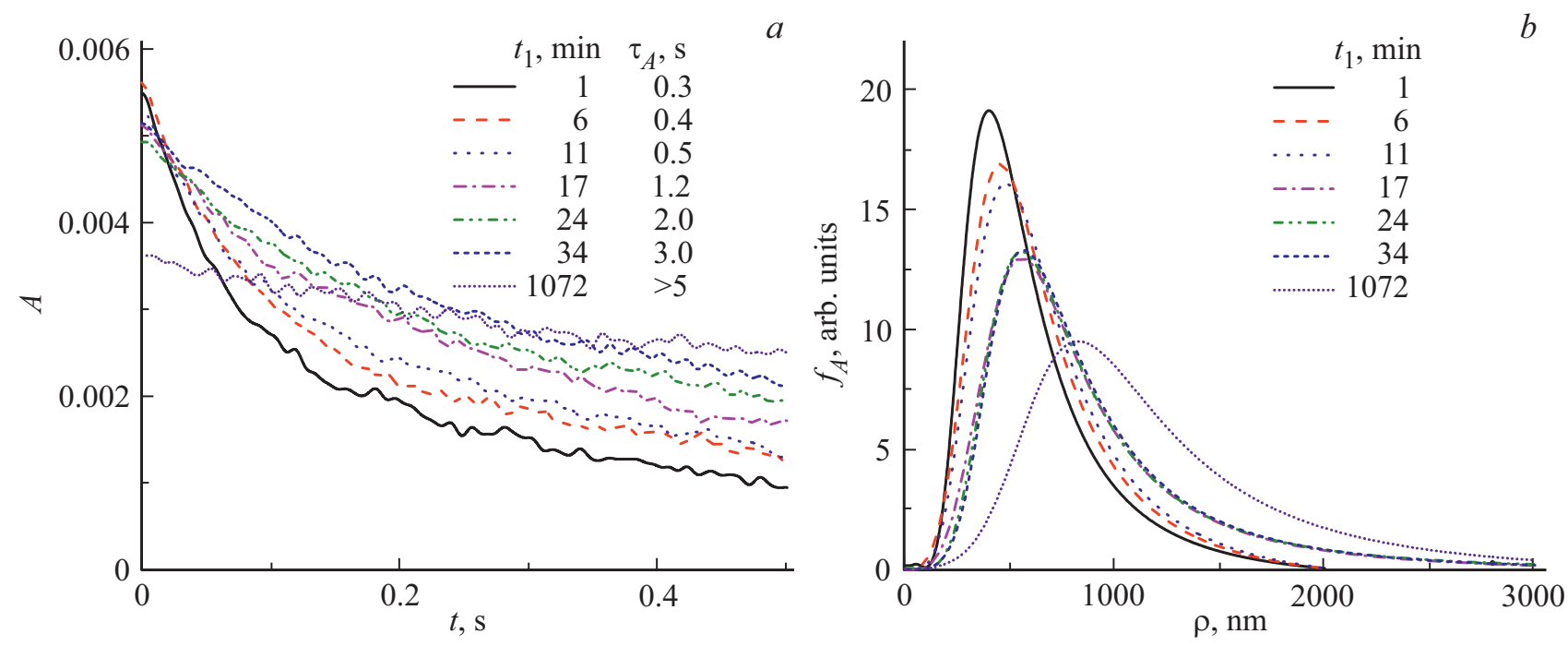

Рис. 8. Изменение со временем $t_{1}$ релаксационной зависимости $A(t)(a)$ и функции распределения $f_{A}(\rho)(b)$ для дисперсной системы графита с концентрацией электролита $\mathrm{AlCl}_{3}$, соответствующей изоэлектрической точке.

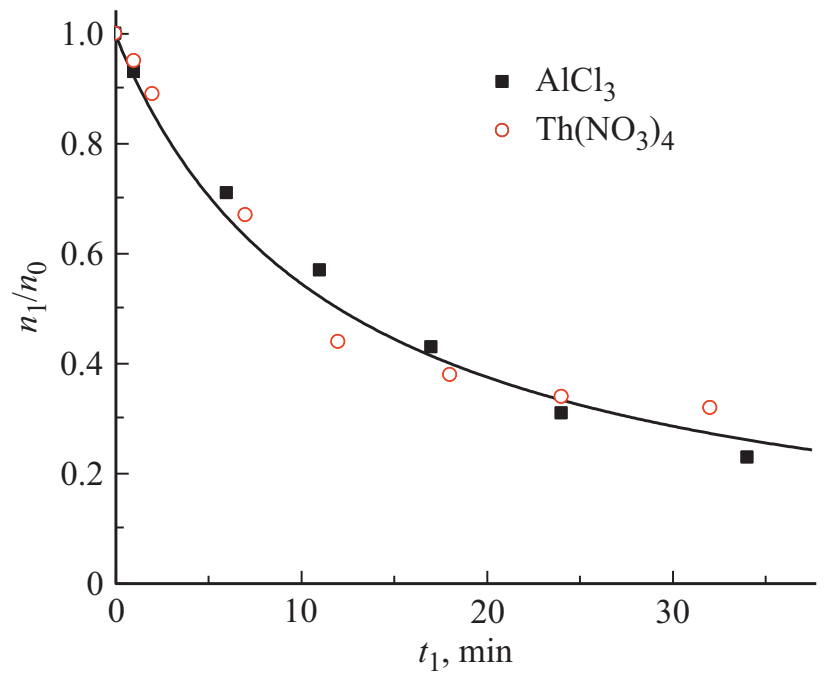

Рис. 9. Теоретическая (сплошная линия) и экспериментальные зависимости $n_{1} / n_{0}$ от $t_{1}$ для дисперсной системы графита в электролитах $\mathrm{AlCl}_{3}$ и $\mathrm{Th}\left(\mathrm{NO}_{3}\right)_{4}$ с концентрациями, соответствующими изоэлектрической точке.

в изоэлектрической точке, представлены на рис. 9. Как видно из рисунка, теоретическая зависимость $n_{1}\left(t_{1}\right) / n_{0}$, определенная при $T_{C}=12.5 \mathrm{~min}$, хорошо согласуется с экспериментальными зависимостями для обоих электролитов. Совпадение экспериментальных зависимостей $n_{1}\left(t_{1}\right) / n_{0}$ соответствует тому, что скорость коагуляции зависит от электрокинетического потенциала частиц и не зависит от качественного различия ионов в электролитах $\mathrm{AlCl}_{3}$ и $\mathrm{Th}\left(\mathrm{NO}_{3}\right)_{4}$.

Значения зависимостей $f_{N}(r)$, представленных на рис. 6 и 7, при $r>300 \mathrm{~nm}$ не уменьшаются при возрастании $t_{1}$, а увеличиваются. Область изменения $r$ практически удваивается. Это свидетельствует об об- разовании парных агрегатов, вносящих вклад в дихроизм $N$. Следует заметить, что в начале коагуляции $\left(t_{1}<2 T_{C}\right)$ значение $N(0)$ меняется не более чем на $10 \%$, но существенно увеличивается время релаксации $\tau_{N}$, что свидетельствует о том, что образование парного агрегата из малых частиц слабо меняет их вклад в дихроизм. Как можно видеть, при больших значениях времени $t_{1}$ величина $N(0)$ уменьшается, что связано с уменьшением объемной доли частиц, вносящих вклад в $N$, по причине образования более крупных агрегатов и седиментации. Крупные частицы, доля которых мала, слабо влияют на дихроизм, а основной вклад они вносят в эффект $A$. Как можно видеть из рис. 8 , изменение $A(t)$ и $f_{A}(\rho)$ в процессе коагуляции аналогично изменению зависимостей $N(t)$ и $f_{N}(r)$. Это подтверждает тот факт, что механизм и время коагуляции крупных и мелких частиц графита близки, что согласуется с теорией быстрой коагуляции.

\section{Заключение}

Электрооптический метод обладает широкими возможностями исследования кинетики коагуляции жидких нанодисперсных систем и особенностей образования агрегатов из частиц. Часть этих возможностей продемонстрирована на примере системы, состоящей из частиц графита, взвешенных в водных электролитах $\mathrm{AlCl}_{3}$ и $\mathrm{Th}\left(\mathrm{NO}_{3}\right)_{4}$. Изменение электрооптических свойств, в частности кривых релаксации электрооптического эффекта, позволяет надежно исследовать кинетику коагуляции дисперсных систем, содержащих частицы графита. Проведенные исследования показали, что эти системы устойчивы в широкой области концентраций электролитов. Зоне коагуляции отвечает низкий электрокинетический потенциал частиц, который не превышает $5 \mathrm{mV}$. 
В изоэлектрической точке, для которой можно не учитывать электростатическое расталкивание частиц, теория быстрой коагуляции хорошо согласуется с наблюдаемыми процессами образования агрегатов из частиц графита. Проведенные исследования подтверждают вывод о том, что на процесс образования агрегатов из частиц с лиофобной поверхностью основное влияние оказывает валентность ионов, определяющих заряд поверхности.

Часть исследований была выполнена в „Междисциплинарном ресурсном центре по направлению „нанотехнологии“, в центре „рентгенодифракционные методы исследования“ и „центре диагностики функциональных материалов для медицины, фармакологии и наноэлектроники“ ресурсного парка Санкт-Петербургского государственного университета.

\section{Список литературы}

[1] Russel W.B., Saville D.A., Schowalter W.R. Colloidal Dispersions. Cambridge University Press Cambridge. 1989. 544 p. doi $10.1017 / \mathrm{CBO} 9780511608810$

[2] Elimelech M., Gregory J., Jia X., Williams R.A. Particle Deposition and Aggregation: Measurement, Modeling, and Simulation. Butterworth-Heinemann Ltd. Oxford. 1995. 448 p. doi 10.1016/B978-0-7506-7024-1.X5000-6

[3] Xu S., Sun Z. // Soft Matter. 2011. V. 7. P. 11298. doi 10.1039/C1SM06237A

[4] Ntampou X., Zouboulis A.I., Samaras P. // Chemosphere. 2006. V. 62. N 5. P. 722. doi 10.1016/j.chemosphere.2005.04.067

[5] Trefalt G., Szilagyi I., Borkovec M. Measuring particle aggregation rates by light scattering. [Электронный ресурс] Режим доступа: http://citeseerx.ist.psu.edu/viewdoc/download? $\mathrm{doi}=10.1 .1 .722 .7171 \& \mathrm{rep}=$ rep1\&type $=\mathrm{pdf}$

[6] Holthoff H., Egelhaaf S.U., Borkovec M., Schurtenberger P., Sticher H. // Langmuir. 1996. V. 12. P. 5541. doi 10.1021/la960326e

[7] Holthoff H., Schmitt A., Fernandez-Barbero A., Borkovec M., Cabrerizo-Vilchez M.A., Schurtenberger P., Hidalgo-Alvarez $R$. // J. Colloid Interface Sci. 1997. V. 192. P. 463. doi $10.1006 /$ jcis. 1997.5022

[8] Li X., May K.Y., Ni X., He C., Leong K.W., Li J. // J. Phys. Chem. B. 2006. V. 110. P. 5920. doi 10.1021/jp057004g

[9] Bloomfield V.A. // Biopolymers. 2000. V. 54. P. 168. doi 10.1002/1097-0282(200009)54:3<168::AID-BIP20> 3.0.CO; 2-9

[10] Войтылов В.В., Трусов А.А., Спартаков А.А. // Опт. и спектр. 1978. Т. 44. № 4. С. 606.

[11] Klemeshev S.A., Petrov M.P., Trusov A.A., Voitylov A.V. // J. Phys. Condens. Matter. 2010. V. 22. P. 494106. doi 10.1088/0953-8984/22/49/494106

[12] Бабаджсаняни, Л.К., Войтылов А.В., Войтылов В.В., Трусов А.A. // Высокомолекулярные соединения. Серия С. 2010. T. 52-7. C. 1329. Babadzhanyants L.K., Voitylov A.V., Voitylov V.V., Trusov A.A. // Polymer Sci. Ser. C. 2010. V. 52. N 7. P. 93. doi 10.1134/S181123821001011X

[13] Muller H. // J. Opt. Soc. Amer. 1941. V. 31. P. 286. doi 10.1364/JOSA.31.000286

[14] Цветков В.Н. Жесткоцепные полимерные молекулы. Л.: Наука, 1986. $380 \mathrm{c.}$
[15] García-Valenzuela A., Barrera R.G., Sanchez-Pérez C., Reyes-Coronado A., Méndez E.R. // Opt. Express. 2005. V. 13. P. 6723. doi 10.1364/OPEX.13.006723

[16] Шиольский Э.В. // УФН. 1945. Т. 27. С. 97. doi 10.3367/UFNr.0027.194501f.0096

[17] Войтылов В.В., Петров М.П., Спартаков А.А., Трусов A.A. // Опт. и спектр. 2013. Т. 114. № 4. C. 687; Vojtylov V.V., Petrov M.P., Spartakov A.A., Trusov A.A. // Opt. Spectrosc. 2013. V. 114. N 4. P. 630-638. doi 10.1134/S0030400X13030272

[18] Толстой Н.А., Феобилов П.П. // ДАН СССР. 1949. Т. 66. C. 617.

[19] Stoylov S., Sheludko A., Chernev R. // Godishnik Sofiskiya Univ., Khim Fak. 1963/64. V. 58. P. 113.

[20] Петров М.П., Войтылов В.В., Клемешев С.А. // Опт. и спектр. 2011. Т. 111. № 5. С. 871; Petrov M.P., Voitylov V.V., Klemeshev S.A., Trusov A.A. // Opt. Spectrosc. 2011. V. 111. N 5. P. 832. doi $10.1134 / \mathrm{S} 0030400 \mathrm{X} 11090207$

[21] Arenas-Guerrero P., Jiménez M.L., Scott K., Donovan K.J. // Carbon. 2018. V. 126. P. 77. doi 10.1016/j.carbon.2017.10.001

[22] Hong S.H., Shen T.Z., Song J.K. // J. Phys. Chem. C. 2014. V. 118. P. 26304. doi 10.1021/jp504892s

[23] Klemeshev S.A., Petrov M.P., Trusov A.A., Vojtylov V.V. // Colloids and Surfaces. A. 2012. V. 400. P. 52. doi 10.1016/j.colsurfa.2012.02.045

[24] Войтылов В.В., Иващенко П.И., Трусов А.А., Алтухов И.В. // Опт. и спектр. 2009. Т. 107. № 5. C. 757; Voitylov V.V., Ivashchenko P.I., Trusov A.A., Altukhov I.V. // Opt. Spectrosc. 2009. V. 107. N 5. P. 717. doi 10.1134/S0030400X09110083

[25] Fredericq E.,Houssier C. Electric Dichroism and Electric Birefringence. Oxford: Clarenden Press, 1973. 219 p.

[26] Дерягин Б.В. Теория устойчивости коллоидов и тонких пленок. М.: Наука, 1986. 206 с.

[27] Verwey E.J.W., Overbeek J.Th.G. Theory of Stability of Lyophobic Colloids. Amsterdam: Elsevier, 1948. 205 p.

[28] Карпов С.В., Семина П.Н. // Коллоидный журнал. 2012. T. 74. № 3. C. 313; Karpov S.V., Semina P.N. // Colloid J. 2012. V. 74. N 3. P. 295. doi 10.1134/S1061933X12030040

[29] van de Hulst H.C. Light scattering by small particles. Dover Publications, Corrected Edition, 1981. 496 р. Перевод: ван де Хюлст Г. Рассеяние света малыми частицами. М.: Иностр. лит-ра, 1961. 536 с.

[30] Perrin F. // J. Phys. Rad. 1934. V. 5. P. 497. doi 10.1051/jphysrad:01934005010049700

[31] Вукс М.Ф. Электрические и оптические свойства молекул и конденсированных сред. Л.: ЛГУ, 1984. 336 с.

[32] Pecora R. (Editor). Dynamic light scattering: applications of photon correlation spectroscopy. Springer, 1985.420 p. 